\title{
Asymptotic Analysis of Contention Resolution ALOHA with Replica Concatenation
}

\author{
Talha Akyıldız*, Umut Demirhan ${ }^{\dagger}$, Tolga M. Duman* \\ ${ }^{*}$ EEE Dept., Bilkent University, Ankara, Turkey \\ ${ }^{\dagger}$ School of ECEE, Arizona State University, Tempe, AZ \\ Email: \{akyildiz,duman\}@bilkent.edu.tr, udemirhan@asu.edu
}

\begin{abstract}
In this paper, we present an asymptotic performance analysis of contention resolution ALOHA (CRA) on the collision channel for both regular and irregular repetition rates. In addition, we consider an improvement to CRA by merging the clean parts of replicas in partial collisions and extend our analysis to this scenario. Specific designs of repetition distributions based on the new analysis show that the optimized solutions for irregular repetition slotted ALOHA (IRSA) perform well in both CRA and the enhanced scheme.

Index Terms-Contention Resolution ALOHA, asymptotic analysis, successive interference cancellation.
\end{abstract}

\section{INTRODUCTION}

With the foreseen proliferation of machine type devices (MTDs), massive machine-to-machine (M2M) communications and random access (RA) schemes have recently attracted great interest. In massive $\mathrm{M} 2 \mathrm{M}$ communications, the number of users is extremely high, however, each user is only sporadically active, hence the number of active users at a certain time is limited. In such scenarios, random access approaches promise convenient and elegant solutions, especially with the utilization of successive interference cancellation (SIC) techniques [1]. In this setting, while slotted RA schemes provide better performance by taking the advantage of slot level synchronization, design and analysis of advanced unslotted schemes are also highly appealing due to the increased synchronization challenges for the slotted case.

Advancements made to the predecessor RA schemes, namely ALOHA [2] and slotted ALOHA [3], with diversity [4] and SIC provide enormous improvements on the maximum throughput. For instance, contention resolution diversity slotted ALOHA (CRDSA) [1] is a slot-frame synchronous scheme where each user sends two packet replicas in each frame and the received packets are resolved via SIC among different slots, resulting in a maximum throughput of 0.55 packets per slot (as opposed to 0.36 for slotted ALOHA). Liva, in [5], proposes irregular repetition slotted ALOHA (IRSA), which generalizes CRDSA by varying the number of replicas, and optimizes the replica distributions via a graphbased asymptotic analysis to obtain (asymptotic) throughputs approaching 1. A more generic scheme, called coded slotted ALOHA [6], and many extensions on these schemes for different scenarios are also proposed (e.g., [7]-[11]).

Departing from the slotted ALOHA variants, to alleviate the strict synchronization requirements, the work [12] proposes contention resolution ALOHA (CRA), which can be consid- ered as an unslotted frame synchronous adaptation of CRDSA where each user sends a fixed number of replicas and collisions are resolved through SIC. It also incorporates strong forward error correction (FEC) codes to resolve the collided packets on the physical layer and demonstrates the resulting performance via simulations. Enhanced CRA (ECRA) is proposed in [13] extending the CRA by merging parts of the packets with minimum interference (or no collision whenever possible) to utilize the FEC codes more efficiently. The authors in [14] propose a fully asynchronous implementation of CRDSA (coupled with an approximate analysis) resolving the collisions with FEC based on the signal-to-interference noise ratio (SINR) of the received packets. In [15], an approximate performance bound for low channel loads with simulation results for frameless ECRA adopting different combining techniques is given.

Although there are numerous works for asynchronous diversity ALOHA schemes, to the best of our knowledge, there is no asymptotic analysis for CRA over the collision channel in the present literature. With this motivation, we develop such an analysis assuming that collisions are resolved through SIC. Initially we consider regular repetition rates, and then we generalize our approach to irregular repetition distributions. We refer to the latter scheme as irregular repetition ALOHA (IRA). Via the proposed analysis, we observe that introducing irregular repetition rates offers higher asymptotic throughputs. We also consider concatenation of the clean parts of two of the replicas in partial collisions if the packet is resolvable, named CRA/IRA with two replica concatenation (CRARC/IRARC) to improve the system throughput. We extend our earlier analysis, which is amenable for further extensions, to this scheme as well. In addition, we verify the accuracy of the developed asymptotic analytical results through finite length simulations.

The paper is organized as follows. In Section II, we describe the system model. The asymptotic analyses of CRA and IRA are developed in Section III. In Section IV, the analysis is extended to CRA/IRA with two replica concatenation. Optimized repetition distributions and numerical results are given in Section V. Finally, Section VI presents our conclusions.

\section{SYSTEM MODEL}

We consider a slot-asynchronous random access scheme with the motivation that such schemes can allow users to transmit their packets at arbitrary time instants within a frame, and hence, they remove the stringent slot synchronization requirements among users. We consider CRA/IRA and 
CRARC/IRARC. In both schemes, users transmit their packets according to a certain repetition rate at different time instants during the frame. The repetition rate might be regular, i.e., each user may transmit $d$ copies of their packets (replicas), or it can be drawn from a certain probability mass function (PMF) as in IRSA. We assume that all the packet replicas have equal length $\tau$. The traffic generated by users, namely normalized load is given by $G=\frac{\tau \cdot n}{T_{f}}$ where $n$ is the number of users and $T_{f}$ denotes the frame length. We also define the load generated by multiple packet replicas, i.e., the physical load, denoted as $\mu$, as the expected number of packet replicas in an interval of length $\tau$, i.e., $\mu=d_{\text {avg }} \cdot G$ where $d_{\text {avg }}$ is the expected number of packet replicas per user. Assuming a large frame length, we model the replica arrival times as a Poisson process with mean $\mu$.

We assume a multiple access collision channel, typically adopted in the analysis of RA schemes [5], where the receiver is able to detect whether a packet experiences collision or not. Collisions are fully destructive and if a packet experiences interference, it cannot be resolved correctly. Otherwise, it is always decoded successfully. We also assume that the receiver is able to execute SIC. Thus, after decoding of a replica of a certain packet, the receiver can remove all of its replicas in the frame. For the enhanced scheme with replica concatenation, we exploit the clean parts of two collided replicas to obtain a complete clean packet. Hence, during the iterative decoding process, the receiver also checks for concatenation of overlapped replicas.

Finally, we define the packet loss rate $(P L R)$ as the percentage of users (on average) not being successfully decoded by the receiver after all the iterations are completed, and the normalized throughput, $T$, as the expected number of correctly decoded user packets in an interval of length $\tau$.

\section{Asymptotic ANALYsis of CRA/IRA}

We now present an analysis for CRA and IRA. We consider infinite frame lengths $\left(T_{f} \rightarrow \infty\right)$ allowing us to examine each replica separately. In the asymptotic setting, the replicas are statistically independent from each other and have identical statistics (including the success and failure probabilities through the SIC iterations). Hence, determining the behavior of a sample replica is sufficient to analyze the entire scheme.

\section{A. Regular Repetition Rates (CRA)}

In this case, each user's packet is transmitted via $d$ repetitions. Consider a specific packet replica and define $A_{i}$ as the event that the replica is not resolvable by iteration $i$. We denote the probability of this event by $q_{i}$, i.e., $P\left(A_{i}\right)=q_{i}$. We also define the complementary event, $\overline{A_{i}}$, with probability $p_{i}$, that denotes the probability of the specific replica (hence the packet) being successfully decoded by the end of iteration $i$. Clearly, $p_{i}=1-q_{i}, P\left(A_{0}\right)=1$ (prior to the iterations, none of the replicas are resolvable) and $A_{1} \supseteq A_{2} \supseteq \cdots \supseteq A_{i_{\max }}$ where $i_{\max }$ is the maximum number of iterations by noting that if a packet is not correctly received by iteration $j$, it means that it is not successfully decoded at iteration $i, i<j$, either.
The length of vulnerable period of a replica is $2 \tau$, i.e., the replicas that arrive in a specific interval of length of $2 \tau$ collide with the reference replica. The probability of $k$ replica arrivals inside an interval of length $2 \tau$ has a Poisson distribution with density $2 \mu$, that is,

$$
P(k \text { replicas in an interval of length } 2 \tau)=\frac{e^{-2 \mu}(2 \mu)^{k}}{k !} .
$$

At the first iteration, none of the replicas are resolved, hence for successful decoding of the chosen replica, there should not be any replica arrival inside the interval of length $2 \tau$, i.e.,

$$
p_{1}=e^{-2 \mu} \text { and } q_{1}=1-e^{-2 \mu} .
$$

Consider a specific packet replica after the execution of the first iteration. In order to resolve this replica, there should not be any undecoded replicas inside its vulnerable period. Hence, the remaining load, which is the expected arrival rate of unresolved replicas, with respect to a replica, can be denoted as $\mu_{2}=\mu q_{1}^{d-1}$, i.e., the load is scaled with the probability of an arbitrary packet not being resolved in the first iteration ${ }^{1}$. Hence, by using the remaining load, we write $p_{2}$ and $q_{2}$ as,

$$
p_{2}=e^{-2 \mu_{2}} \text { and } q_{2}=1-e^{-2 \mu_{2}} .
$$

To clarify, we now compute $p_{2}$ and $q_{2}$ for a specific replica explicitly. With $k$ replicas inside its vulnerable period, $2 \tau$, either there are no unresolved replicas, i.e., all the $k$ replicas are decoded at iteration 1 , and the specific replica can be resolved, or there is at least one unresolved replica and the specific replica cannot be resolved. Since each of the $k$ replicas has a probability of $\left(1-q_{1}^{d-1}\right)$ being resolved, the probability of all of the $k$ replicas being decoded is written as $\left(1-q_{1}^{d-1}\right)^{k}$. Thus, by applying the total probability theorem, we obtain

$$
\begin{aligned}
p_{2} & =\sum_{k=0}^{\infty} \frac{e^{-2 \mu}(2 \mu)^{k}}{k !}\left(1-q_{1}^{d-1}\right)^{k} \\
& =e^{-2 \mu_{2}},
\end{aligned}
$$

and $q_{2}=1-e^{-2 \mu_{2}}$ by using $\mu_{2}=\mu q_{1}^{d-1}$. The remaining load at iteration 3 corresponds to the load generated by replicas of the unresolved packets at iteration 2, therefore $\mu_{3}=\mu q_{2}^{d-1}=\mu_{2}\left(\frac{q_{2}}{q_{1}}\right)^{d-1}$. For iteration $i$, we can write $p_{i}$ and $q_{i}$ with respect to the remaining load $\mu_{i}=\mu q_{i-1}^{d-1}$ by considering the conditional probability of a packet not being decoded at iteration $i-1$ given that it is not resolved at iteration $i-2$ (notice that if a packet is not decoded at iteration $i-1$, it is certainly not decoded at iteration $i-2$ either) as follows:

$$
P\left(A_{i-1} \mid A_{i-2}\right)=\frac{P\left(A_{i-1}\right)}{P\left(A_{i-2}\right)}=\frac{q_{i-1}}{q_{i-2}} .
$$

That is, by using $\mu_{i}=\mu_{i-1}\left(\frac{q_{i-1}}{q_{i-2}}\right)^{d-1}$, we obtain

$$
\begin{aligned}
p_{i} & =\sum_{k=0}^{\infty} \frac{e^{-2 \mu_{i-1}}\left(2 \mu_{i-1}\right)^{k}}{k !}\left(1-\left(\frac{q_{i-1}}{q_{i-2}}\right)^{d-1}\right)^{k} \\
& =e^{-2 \mu_{i}},
\end{aligned}
$$

and $q_{i}=1-e^{-2 \mu_{i}}$.

\footnotetext{
${ }^{1}$ We will refer to $\mu_{i}$ as the remaining load at iteration $i$.
} 


\section{B. Irregular Repetition Rates (IRA)}

We now consider the scenario where the number of replicas are drawn from an arbitrary PMF, instead of being picked as constant $d$. We define $\Lambda(x)$ as the user degree distribution, i.e.,

$$
\Lambda(x) \triangleq \sum_{d=1}^{d_{\max }} \Lambda_{d} x^{d},
$$

where $\Lambda_{d}$ denotes the probability of a user transmitting $d$ replicas and $d_{\max }$ is the maximum number of replicas. Average packet repetition rate can be found as $\Lambda^{\prime}(1)=\sum_{d} \Lambda_{d} d$.

In order to extend the analysis in the previous subsection to this case, we again examine the receiver operations on a specific packet replica. We first compute the probability of a randomly selected replica belonging to a user with a repetition rate of $d$ as

$$
\lambda_{d}=\frac{\Lambda_{d} d}{\sum_{d=1}^{d_{\max }} \Lambda_{d} d} .
$$

We also define the replica degree distribution in polynomial form, $\lambda(x)$, as

$$
\lambda(x) \triangleq \sum_{d=1}^{d_{\max }} \lambda_{d} x^{d-1} \text { where } \lambda(x)=\frac{\Lambda^{\prime}(x)}{\Lambda^{\prime}(1)} .
$$

We update the remaining load formulation, $\mu_{i}=\mu q_{i-1}^{d-1}$, by averaging over the replica degree distribution as

$$
\mu_{i}=\mu \sum_{d=1}^{d_{\max }} \lambda_{d}\left(q_{i-1}^{d-1}\right)=\mu \lambda\left(q_{i-1}\right),
$$

where $\mu=\Lambda^{\prime}(1) \cdot G$. The remaining load can also be expressed in terms of $G$ as, $\mu_{i}=\Lambda^{\prime}(1) \cdot G \cdot \lambda\left(q_{i-1}\right)$, and we can rewrite $p_{i}$ and $q_{i}$ in a generic form as

$$
p_{i}=e^{-2 \mu_{i}} \text { and } q_{i}=1-e^{-2 \mu_{i}} .
$$

Finally, recalling the definitions of the packet loss rate and the normalized throughput, we write $P L R=\Lambda\left(q_{i_{\max }}\right)$ and $T=(1-P L R) G$. We emphasize that these expressions are valid for both regular and irregular repetition scenarios.

\section{CRA/IRA WITH Two REPLICA CONCATENATION (CRARC/IRARC)}

We now consider an extended version of CRA/IRA with SIC by concatenation of two overlapped packet replicas to build an interference free packet from the clean parts of collided versions. Our aim is to benefit from these partial collisions caused by asynchronism, which do not exist in the slot synchronous schemes. We limit the number of replicas whose clean parts are combined to only two in order to keep the algorithm simple, however, we also examine the effect of this simplification in the numerical results section.

\section{A. Preliminaries}

There are four possible cases that a packet replica (with some clean portion) may encounter as illustrated in Fig. 1. To explain, we divide the vulnerable period of the replica under consideration into two equal length intervals as $(a-\tau, a)$ and $(a, a+\tau)$ (where $a$ is the transmission start time of the packet). For case I, the packet is collided only from the lefthand side meaning that there are colliding replicas starting in $(a-\tau, a)$ only. A similar argument holds for the packet that is only collided from the right-hand side (case II). For case III, the packet is collided from both sides at the same time. In addition to these three cases, for case IV, the packet is completely interference free.

We now determine the amount of collisions from the lefthand and right-hand sides. We define two events $C_{k}^{-}$and $C_{l}^{+}$ to represent $k$ and $l$ colliding replica arrivals in the intervals $(a-\tau, a)$ and $(a, a+\tau)$, respectively. Therefore,

$$
P\left(C_{k}^{-}\right)=\frac{e^{-\mu} \mu^{k}}{k !}, \quad P\left(C_{l}^{+}\right)=\frac{e^{-\mu} \mu^{l}}{l !},
$$

as the arrivals follow a Poisson process with density $\mu$. For Poisson arrivals, conditioned on the number of arrivals in a given interval, the arrival time of the replicas are independent and identically distributed (i.i.d.) uniform random variables within their respective intervals. Hence we condition on the events $C_{k}^{-}$and $C_{l}^{+}$, denote the arrival times of the replicas in $(a-\tau, a)$ and $(a, a+\tau)$ as $U_{1}^{-}, U_{2}^{-}, \ldots, U_{k}^{-}$and $U_{1}^{+}, U_{2}^{+}, \ldots, U_{l}^{+}$, respectively, and take $\tau=1$ for simplicity of notation, we define random variables $X, Y \in[0,1]$ to denote the maximum amount of collision from the left-hand and the right-hand sides, i.e.,

$$
X=\max _{1 \leq i \leq k} U_{i}^{-}, \quad Y=1-\min _{1 \leq j \leq l} U_{j}^{+} .
$$

The cumulative distribution function (CDF) and probability density function (PDF) of $X$ and $Y$ conditioned on $C_{k}^{-}$and $C_{l}^{+}$are: $F_{X}\left(x \mid C_{k}^{-}\right)=x^{k}, F_{Y}\left(y \mid C_{l}^{+}\right)=y^{l}, f_{X}\left(x \mid C_{k}^{-}\right)=$ $k x^{k-1}$, and $f_{Y}\left(y \mid C_{l}^{+}\right)=l y^{l-1}, x, y \in[0,1]$. Note that conditional PDFs and CDFs of $X$ and $Y$ are identical, and by using the total probability theorem, we can write

$$
\begin{aligned}
F_{X}(x) & =\sum_{k=0}^{\infty} P\left(C_{k}^{-}\right) F_{X}\left(x \mid C_{k}^{-}\right) \\
& =e^{-\mu(1-x)}, \text { for } x \in[0,1] .
\end{aligned}
$$

Hence $f_{X}(x)=e^{-\mu} \delta(x)+\mu e^{-\mu(1-x)}(u(x)-u(x-1))$ where $u(x)$ is the unit step function, i.e., $X$ is a mixed random variable with a mass point at $x=0$. Note that for the asymptotic analysis, we utilize the continuous part of $X$. Hence, the conditional PDF and CDF defined as $f_{X \mid B}(x)=\frac{\mu e^{-\mu(1-x)}}{1-e^{-\mu}}$ and $F_{X \mid B}(x)=\frac{e^{-\mu(1-x)}-e^{-\mu}}{1-e^{-\mu}}, x \in(0,1]$, with $B=\{X \neq 0\}$ are also needed.

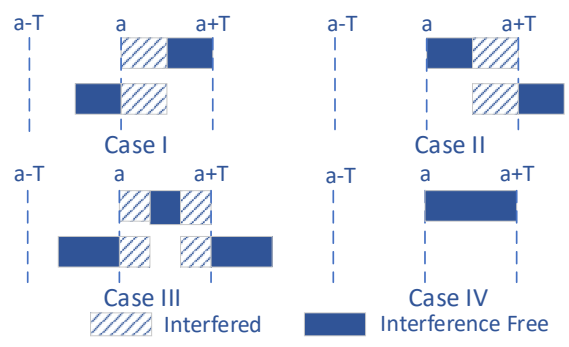

Fig. 1. Possible collision scenarios for a packet replica with some clean part. 


\section{B. Asymptotic Analysis}

We now provide an asymptotic analysis of CRA/IRA with two replica concatenation. For the analysis, we only need the additional resolvability probability (due to the packets that cannot be resolved by CRA/IRA alone). Hence, the objective is to find the successful concatenation probability of the unresolved packets using the clean portions of colliding replicas. Note that using only two partially colliding packets for concatenation simplifies our analysis since, in this case, if a replica is collided from both sides (case III), it is not utilized to recover a packet. Hence, these two replicas need to have collisions only from the left- and right-hand sides (cases III), respectively. Consider two replicas which belong to case I and II, respectively. Free parts of these replicas are in the intervals $\left[0,1-Y_{1}\right]$ and $\left[X_{2}, 1\right]$. The successful recovery, i.e., $[0,1]=\left[0,1-Y_{1}\right] \cup\left[X_{2}, 1\right]$, is possible only if $X_{2} \leqslant 1-Y_{1}$.

Let us consider a user with $d$ packet replicas. We denote the number of replicas experiencing case-I, case-II and case-III by $k, n$ and $d-n-k$ respectively. The selection of replicas can be made in $\left(\begin{array}{c}d \\ n, k\end{array}\right)=\frac{d !}{n ! k !(d-n-k) !}$ different ways. With $g_{n, k, d, \mu}$ denoting the probability of $k, n$ and $d-n-k$ packets belonging to case-I, case-II and case-III respectively, we write

$$
\begin{aligned}
g_{n, k, d, \mu}= & \left(\begin{array}{c}
d \\
n, k
\end{array}\right) \prod_{l=1}^{n} P\left(X_{l}=0, Y_{l} \neq 0\right) \prod_{m=n+1}^{n+k} P\left(X_{m} \neq 0, Y_{m}=0\right) \\
& \cdot \prod_{s=n+k+1}^{d} P\left(X_{s} \neq 0, Y_{s} \neq 0\right) .
\end{aligned}
$$

With this definition, we first search for the replica with the largest clean part from the left-hand side that belong to caseII, and then look for a second replica with the smallest collided part from the left-hand from case-I that we can concatenate with the first one. The amount of largest clean part from the left-hand among the $n$ replicas is denoted by $Z_{n}$, i.e., $Z_{n}=$ $\max _{1 \leq l \leq l}\left(1-Y_{l}\right)$ and the amount of smallest collided part from the

left-hand among the $k$ replicas is denoted by $W_{k}$, i.e., $W_{k}=$ $\min _{1 \leq m \leq k}\left(X_{n+m}\right)$. Clearly, the successful recovery is possible, as we mentioned earlier, only if $W_{k} \leqslant Z_{n}$.

We then compute the probability of successful concatenation denoted by $p_{n, d, k, \mu}^{c}$ using the above condition and (4):

$$
\begin{aligned}
p_{n, k, d, \mu}^{c}= & \left(\begin{array}{c}
d \\
n, k
\end{array}\right) P\left(D_{n}\right) P\left(E_{k}\right) \prod_{l=1}^{n} P\left(X_{l}=0\right) \prod_{m=n+1}^{n+k} P\left(Y_{m}=0\right) \\
& \cdot P\left(W_{k} \leqslant Z_{n} \mid D_{n}, E_{k}\right) \prod_{s=n+k+1}^{d} P\left(X_{s} \neq 0, Y_{s} \neq 0\right)
\end{aligned}
$$

where $D_{n}=\bigcap_{l=1}^{n}\left\{Y_{l} \neq 0\right\}$ and $E_{k}=\bigcap_{m=1}^{k}\left\{X_{n+m} \neq 0\right\}$. The probability of these events are $P\left(D_{n}\right)=\left(1-e^{-\mu}\right)^{n}$ and $P\left(E_{k}\right)=\left(1-e^{-\mu}\right)^{k}$.

As a next step, we need to calculate the probability $P\left(W_{k} \leqslant Z_{n} \mid D_{n}, E_{k}\right)$. To do this, we first determine the conditional CDF of $W_{k}$ and $Z_{n}$ conditioned on the events $E_{k}$ and $D_{n}$ as

$F_{W_{k} \mid E_{k}}(w)=1-\frac{\left(1-e^{-\mu(1-w)}\right)^{k}}{\left(1-e^{-\mu}\right)^{k}}, F_{Z_{n} \mid D_{n}}(z)=\frac{\left(1-e^{-\mu z}\right)^{n}}{\left(1-e^{-\mu}\right)^{n}}$, for $w, z \in(0,1]$. Using these, we obtain

$$
P\left(W_{k} \leqslant Z_{n} \mid D_{n}, E_{k}\right)=1-\frac{n}{\left(1-e^{-\mu}\right)^{n+k}} \int_{0}^{1-e^{-\mu}}\left(1-\frac{e^{-\mu}}{1-u}\right) u^{n-1} d u,
$$

by simplifying the integral $\int_{0}^{1} F_{W_{k} \mid E_{k}}(z) f_{Z_{n} \mid D_{n}}(z) d z$. The integral in (6) can be written in terms of the Appell hypergeometric function [16] ${ }^{2}$, i.e.,

$$
\int_{0}^{1-e^{-\mu}}\left(1-\frac{e^{-\mu}}{1-u}\right) u^{n-1} d u=\frac{\left(1-e^{-\mu}\right)^{(n+k)}}{n} F_{1}\left(n ; k,-k ; n+1 ; 1-e^{-\mu}, 1\right),
$$

hence

$$
P\left(W_{k} \leqslant Z_{n} \mid D_{n}, E_{k}\right)=1-F_{1}\left(n ; k,-k ; n+1 ; 1-e^{-\mu}, 1\right) .
$$

We can then rewrite $p_{n, k, d, \mu}^{c}$ by plugging (6) into (5) as

$$
\begin{aligned}
p_{n, k, d, \mu}^{c}= & \left(\begin{array}{c}
d \\
n, k
\end{array}\right) e^{-\mu(n+k)}\left(1-e^{-\mu}\right)^{(2 d-n-k)} \\
& \cdot\left(1-F_{1}\left(n ; k,-k ; n+1 ; 1-e^{-\mu}, 1\right)\right) .
\end{aligned}
$$

We also define $p_{d, \mu}^{c}$ as the total successful concatenation probability of a user with $d$ replicas in terms of $p_{n, k, d, \mu}^{c}$ by summing over all possible $n$ and $k$ values, i.e.,

$$
p_{d, \mu}^{c}=\sum_{n=1}^{d-1} \sum_{k=1}^{d-n} p_{n, k, d, \mu}^{c} .
$$

To utilize these derivations in the analysis, we finally update the generic load $\mu$ with the remaining load at iteration $i, \mu_{i}$. For simplicity, we define the notation $p_{d, i}^{c}=p_{d, \mu_{i}}^{c}$ for a user with a repetition rate $d$. We also need to define the probability of successful concatenation from the replica perspective for iteration updates, denoted by $p_{d, i}^{c, r}$, which can be written in terms of $p_{d, i}^{c}$ as $p_{d, i}^{c, r}=\frac{p_{d, i}^{c}}{q_{i}}$ (where $q_{i}$ stands for the failure probability of a packet).

We then specify $p_{i}^{c}$ and $p_{i}^{c, r}$ as the expected success recovery probabilities for irregular repetition rates by averaging $p_{d, i}^{c}$ and $p_{d, i}^{c, r}$ over the user and replica degree probabilities, $\Lambda_{d}$ and $\lambda_{d}$, as

$$
p_{i}^{c}=\sum_{d=1}^{d_{\max }} \Lambda_{d} p_{d, i}^{c}, p_{i}^{c, r}=\sum_{d=1}^{d_{\max }} \lambda_{d} p_{d, i}^{c, r} .
$$

As the last step, we revise the remaining load defined in Section III as $\mu_{i}=\mu \lambda\left(q_{i-1}\right)$. Since, by performing replica concatenation, the receiver is now able to decode some additional packets among the remaining ones with probability $p_{i-1}^{c, r}$ at iteration $i-1$, the remaining load is updated as $\mu\left(\lambda\left(q_{i-1}\right)-p_{i-1}^{c, r}\right)$ for the $i^{\text {th }}$ iteration.

Finally, the $P L R$ updated as $P L R=\Lambda\left(q_{i_{\max }}\right)-p_{i_{\max }}^{c}$ and $T=(1-P L R) G$ in a similar manner to IRA.

\footnotetext{
${ }^{2}$ The Appell hypergeometric function is defined in a generic form as, $F_{1}\left(a ; b_{1}, b_{2} ; c ; x, y\right)=\sum_{m=0}^{\infty} \sum_{n=0}^{\infty}\left(\frac{(a)_{m+n}\left(b_{1}\right)_{m}\left(b_{2}\right)_{n}}{m ! n !(c)_{m+n}}\right)\left(x^{m} y^{n}\right)$. The term $(x)_{n}$ is the Pochhammer symbol given by $(x)_{n}=\frac{\Gamma(x+n)}{\Gamma(x)}$.
} 


\section{NumERICAL RESUltS}

We now present some numerical examples. We first optimize the degree distributions to maximize the asymptotic channel load $G^{*}$ providing a predefined $P L R$ by using differential evolution [17]. Optimized distributions for different maximum repetition rates and regular distribution with repetition rate 2 with their corresponding highest asymptotic channel load, $G^{*}$, for IRSA, IRA and IRARC are given in Table I. We see that the ratio of maximum achievable throughput, $T^{*}$, between IRSA and IRA is 0.5. We note that this relationship is not particular for irregular repetition rates and valid for any user degree distribution under asymptotic settings, again observed through the analysis in Section III. Furthermore, our results show that the optimized distributions of IRSA are also optimal for both IRA and IRARC ${ }^{3}$.

TABLE I

ASYMPTOTIC PERFormanCE OF DisTRIBUtIONS

\begin{tabular}{|c|c|c|c|}
\hline \hline User Degree Distributions $\Lambda(x)$ & CRDSA/IRSA(G*) & CRA/IRA(G*) & CRARC/IRARC(G*) \\
\hline \hline$\Lambda_{2}(x)=x^{2}$ & 0.541 & 0.271 & 0.344 \\
\hline$\Lambda_{4}(x)=0.5102 x^{2}+0.4898 x^{4}$ & 0.868 & 0.434 & 0.543 \\
\hline$\Lambda_{5}(x)=0.5631 x^{2}+0.0436 x^{3}+0.3933 x^{5}$ & 0.898 & 0.449 & 0.561 \\
\hline$\Lambda_{6}(x)=0.5465 x^{2}+0.1623 x^{3}+0.2912 x^{6}$ & 0.915 & 0.457 & 0.573 \\
\hline$\Lambda_{8}(x)=0.5 x^{2}+0.28 x^{3}+0.22 x^{8}$ & 0.938 & 0.469 & 0.59 \\
\hline \hline
\end{tabular}

The above behavior for the maximum achievable throughputs between IRSA and IRA deserves more explanation. Success and failure probabilities at each iteration for IRSA and IRA with the asymptotic analysis can be written as $p_{1, i}=e^{-\mu_{1, i}}, p_{2, i}=e^{-2 \mu_{2, i}}$ and $q_{1, i}=1-e^{-\mu_{1, i}}$, $q_{2, i}=1-e^{-2 \mu_{2, i}}$ and $\mu_{1, i}=\mu \lambda\left(q_{1, i-1}\right), \mu_{2, i}=\mu \lambda\left(q_{2, i-1}\right)$ where $p_{1, i}$ and $q_{1, i}$ are for IRSA, $p_{2, i}$ and $q_{2, i}$ are for IRA. Due to the lack of slot synchronization, vulnerable period of a packet in IRA is doubled compared to IRSA. Noting that for any given user degree distribution, $\Lambda(x)$ and $\lambda(x)$ functions are the same for both cases, for $\mu_{1, i}=2 \mu_{2, i}$, their success and failure probabilities are equal. By using $\mu=\Lambda^{\prime}(1) G$, we can write $G_{1, i}=2 G_{2, i}$. Under the decoding threshold $G^{*}$, the failure probability and also the $P L R$ converge to zero, i.e., $q \rightarrow 0$ and $P L R \rightarrow 0$ since $P L R=\Lambda(q)$. Using $T^{*}=(1-P L R) G^{*}$, we have $T^{*} \rightarrow G^{*}$ and $G_{1, i}^{*}=2 G_{2, i}^{*}$, we observe that $T_{I R S A}^{*}=2 T_{I R A}^{*}$. Therefore, it is not surprising that the throughput with IRA is half of that of IRSA. We note that while our analysis reveals this simple dependence between the throughputs of IRSA and IRA, this observation is not easy to make without the newly developed machinery.

A similar explanation is not straightforward to make for IRARC. Intuitively, we only have a relatively small additional resolution probability because of concatenation that is derived through same degree distributions, which may be the reason for the optimal distributions for IRA to execute well for IRARC as well.

We now perform finite length simulations for both IRA and IRARC. We evaluate various frame lengths, i.e., 100, 200 and $1000 \mathrm{~ms}$, by assuming all replicas have the same length $\tau=$

${ }^{3}$ Differential evolution algorithm does not guarantee a globally optimal solution. There exists other distributions perform slightly better for IRARC, however, we did not include them as the improvements were very minor.
$1 \mathrm{~ms}$. We keep the maximum number of iterations as 20, $i_{\max }=20$, for all the simulations.

We first provide the simulation results of IRA for $\Lambda_{8}(x)$ and CRA for $\Lambda_{2}(x)$ by using the above predefined frame lengths in Fig. 2 along with the corresponding asymptotic analysis results. The fit of the finite length simulations and the asymptotic analysis is clearly seen for both throughput and $P L R$ as the frame length increases for $\Lambda_{8}(x)$. We observe that the maximum achievable throughput, i.e., the decoding threshold is $G^{*}=T^{*}=0.469$. In addition, the irregular distribution $\Lambda_{8}(x)$ enhances the throughput by approximately $71 \%$ compared to the regular distribution $\Lambda_{2}(x)$, asymptotically ${ }^{4}$. Notice that this enhancement is also observed for finite length simulations though the percentage improvement is smaller.
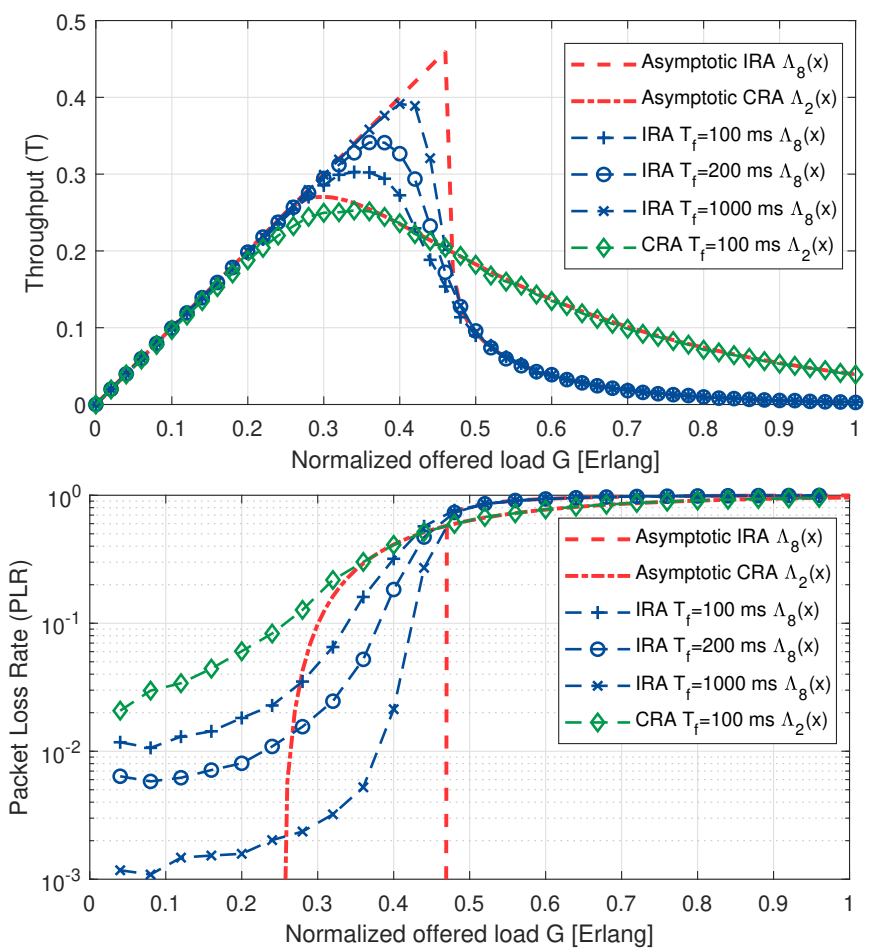

Fig. 2. Asymptotic analysis of CRA/IRA with $\Lambda_{8}(x)$ and $\Lambda_{2}(x)$ for different frame lengths $\left(T_{f}=100,200,1000 \mathrm{~ms}\right), i_{\max }=20$.

We now consider the same set-up with replica concatenation. Fig. 3 illustrates the finite length simulation results for $\Lambda_{8}(x)$ and $\Lambda_{2}(x)$ along with the asymptotic results of IRA with replica concatenation. We observe that the finite length simulation results comply with the asymptotic analysis, and that the decoding threshold $G^{*}$ is increased from 0.469 to 0.59. That is, there is a significant improvement (by about $25 \%$ ) in throughput with replica concatenation. Furthermore, the use of irregular repetition rates offer superior performance as in CRA/IRA.

Finally, Fig. 4 depicts the simulation results of CRA and IRA with 2 and all possible replica concatenation with $T_{f}=$ $200 \mathrm{~ms}$. As noted earlier, introducing 2 replica concatena-

${ }^{4}$ For further comparisons, we also examine the asymptotic performance of regular and irregular distributions by keeping the maximum repetition rate fixed as 4 and 5. For the former, $\Lambda(x)=x^{4}$ and $\Lambda_{4}(x), G^{*}$ is increased from 0.386 to $0.434(\approx 12 \%)$. For the latter, $\Lambda(x)=x^{5}$ and $\Lambda_{5}(x), G^{*}$ is increased from 0.350 to $0.449(\approx 28 \%)$. 

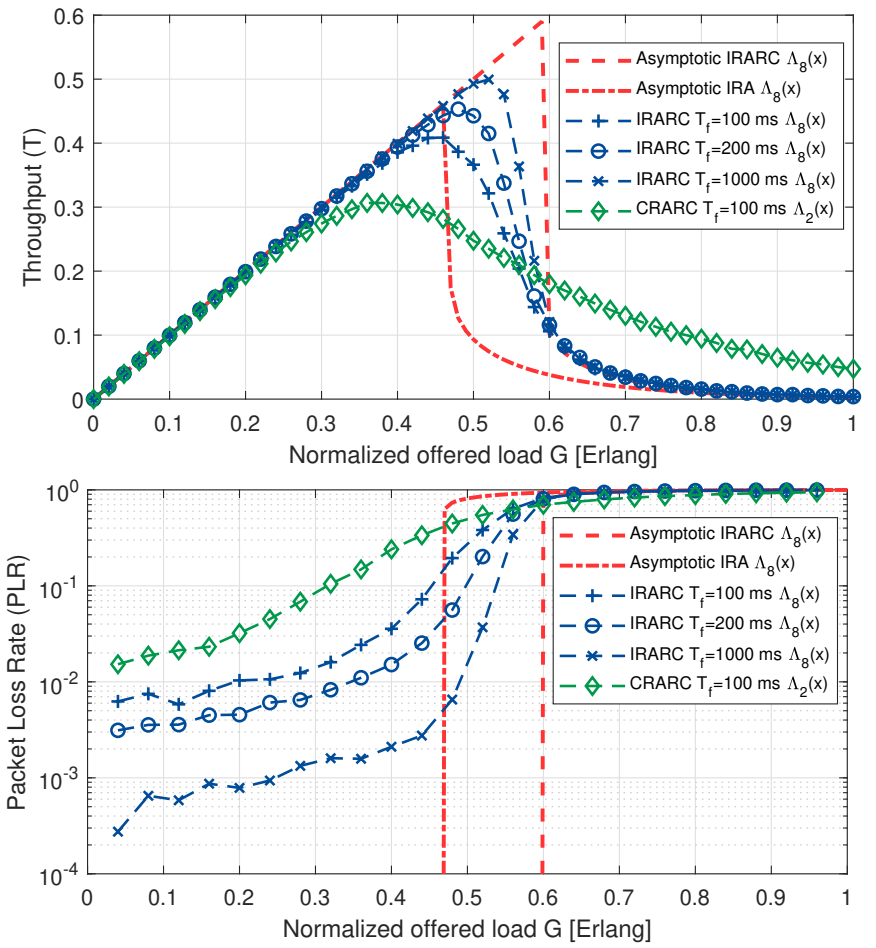

Fig. 3. Asymptotic analysis of IRARC and IRA with $\Lambda_{8}(x)$ for different frame lengths $\left(T_{f}=100,200,1000 \mathrm{~ms}\right), i_{\max }=20$.

tion results in a significant throughput increase compared to CRA/IRA, however, the simulation results show that concatenation of all the replicas performs only slightly better. We explain this as follows, for this specific example, $59 \%$ of the packets are resolved with only 1 replica (no concatenation), $30 \%$ with 2 replica concatenation, and $9 \%$ with 3 replica concatenation (while the remaining percentage is due to concatenation of more replicas) at the specific load value of $G=0.5$. In other words, it may not be worthwhile to concatenate more than two replicas due to additional complexity, as most of the possible gain is already obtained with two. Lastly, in the same plot, we also include supplementary plots for CRA and CRARC with $\Lambda_{2}(x)$ to depict the additional resolvability, and hence, performance gain introduced by replica concatenation.

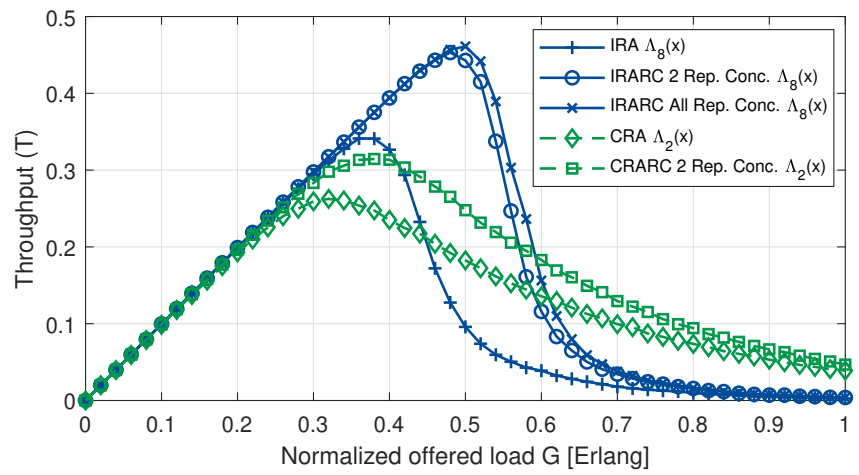

Fig. 4. Simulation results of CRA/IRA and CRARC/IRARC with 2 and all replica concatenation $\Lambda_{8}(x)$ and $\Lambda_{2}(x), T_{f}=200 \mathrm{~ms}, i_{\max }=20$.

\section{CONCLusions}

We have considered slot-asynchronous CRA/IRA and CRA/IRA with two replica concatenation, and developed an asymptotic analysis with a focus on obtaining optimal repetition degree distributions. We first studied the asymptotic analysis of the CRA/IRA and then extended the analysis by introducing two replica concatenation, which provides significant gains in terms of the normalized throughput. The results are corroborated with extensive finite length simulations as well. Finally, we note that the developed analysis techniques are amenable for further extensions; for instance, considering different channel models or slot-asynchronous schemes with power imbalance or different packet durations among users are possible.

\section{REFERENCES}

[1] E. Casini, R. De Gaudenzi, and O. Del Rio Herrero, "Contention resolution diversity slotted ALOHA (CRDSA): An enhanced random access scheme for satellite access packet networks," IEEE Trans. Wirel. Commun., vol. 6, no. 4, pp. 1408-1419, Apr. 2007.

[2] N. Abramson, "The ALOHA system: Another alternative for computer communications," in Proc. Fall Joint Comput. Conf., 1970, pp. 281-285.

[3] L. G. Roberts, "ALOHA packet system with and without slots and capture," ACM SIGCOMM Comput. Commun. Rev., vol. 5, no. 2, pp. 28-42, Apr. 1975.

[4] G. L. Choudhury and S. S. Rappaport, "Diversity ALOHA - A random access scheme for satellite communications," IEEE Trans. Commun., vol. 31, no. 3, pp. 450-457, Mar. 1983.

[5] G. Liva, "Graph-based analysis and optimization of contention resolution diversity slotted ALOHA," IEEE Trans. Commun., vol. 59, no. 2, pp. 477-487, Feb. 2011.

[6] E. Paolini, G. Liva, and M. Chiani, "Coded slotted ALOHA: A graphbased method for uncoordinated multiple access," IEEE Trans. Inf. Theory, vol. 61, no. 12, pp. 6815-6832, Dec. 2015.

[7] C. Stefanovic and P. Popovski, "ALOHA random access that operates as a rateless code," IEEE Trans. Commun., vol. 61, no. 11, pp. 4653-4662, Nov. 2013.

[8] K. R. Narayanan and H. D. Pfister, "Iterative collision resolution for slotted ALOHA: An optimal uncoordinated transmission policy," in Proc. Int. Symp. Turbo Codes Iterative Inf. Process. (ISTC), Gothenburg, Sweden, Aug. 2012, pp. 136-139.

[9] F. Clazzer, E. Paolini, I. Mambelli, and C. Stefanovic, "Irregular repetition slotted ALOHA over the Rayleigh block fading channel with capture," in Proc. IEEE Int. Conf. Commun. (ICC), Paris, France, May 2017.

[10] A. Mengali, R. De Gaudenzi, and P.-D. Arapoglou, "Enhancing the physical layer of contention resolution diversity slotted ALOHA," IEEE Trans. Commun., vol. 65, no. 10, pp. 4295-4308, Oct. 2017.

[11] U. Demirhan and T. M. Duman, "Energy-harvesting irregular repetition slotted aloha with unit-sized battery," in Proc. IEEE Int. Conf. Commun. (ICC), Kansas City, USA, May 2018.

[12] C. Kissling, "Performance enhancements for asynchronous random access protocols over satellite," in Proc. IEEE Int. Conf. Commun. (ICC), Kyoto, Japan, Jun. 2011.

[13] F. Clazzer and C. Kissling, "Enhanced contention resolution ALOHA - ECRA," in Proc. 9th Int. ITG Conf. Syst. Commun. Coding (SCC), vol. 9, Nov. 2013.

[14] R. De Gaudenzi, O. del Rio Herrero, G. Acar, and E. Garrido Barrabes, "Asynchronous contention resolution diversity ALOHA: Making CRDSA truly asynchronous," IEEE Trans. Wirel. Commun., vol. 13, no. 11, pp. 6193-6206, Nov. 2014.

[15] F. Clazzer, C. Kissling, and M. Marchese, "Enhancing contention resolution ALOHA using combining techniques," IEEE Trans. Commun., vol. 66, no. 6, pp. 2576-2587, Jun. 2018.

[16] M. Abramowitz and I. A. Stegun, Handbook of Mathematical Functions with Formulas, Graphs, and Mathematical Tables. New York: Dover, 1964.

[17] R. Storn and K. Price, "Differential evolution - a simple and efficient heuristic for global optimization over continuous spaces," J. Glob. Optim., vol. 11, no. 4, pp. 341-359, Dec. 1997. 